\title{
A Case Study on the Landuse Pattern of Kanyakumari District- Using Gis
}

\author{
E.Vinodhini Shanmugapriya ${ }^{1}$, S.Veda Samhitha ${ }^{2}$, P.Geetha $^{3}$ \\ ${ }^{123}$ Center for Computational Engineering \& Networking (CEN), Amrita School of Engineering, Coimbatore \\ Amrita Vishwa Vidyapeetham,Amrita University, India
}

\begin{abstract}
The fundamental pattern of the land use is the exactness in vegetation strategies. This study focus on the landcover patterns of Kanyakumari district using geographical information system.The evolution of landuse patterns are made using landsat imagery. The variations of vegetation index are determined using ArcGIS softwareThe key advancement in implementing this study is to give brief information about the aerial land classification and human activities over the region of interest. Geo-Satellite information about vegetation landcover helps us to be more proficient in fragmenting areas. The end goal is to decide the type of field patterns and an appropriate area for profitable utilization of land cover features which leads to efficient vegetation.
\end{abstract}

Keywords: ArcGIS; Landsat imagery; Landuse pattern; Remote Sensing; NDVI

\section{Introduction}

Remote Sensing, Satellite Imagery and Geographic system (GIS) are currently providing efficient trending technology for the advancement in system management. The acquisition of perceived information facilitates the synoptic analyses of our geographical system operation thus provides an alternate to the regional and international scales over time, such perceived and processed information additionally offers a very important sink between localized and intensive ecological analysis over regional, national and international conservation management of bio- diversity. The Land use forms a crucial part in the development and dependability of the nation's growth analysis. To understand the durative improvement of vegetation and other land use pattern the analysis started to consider the new cultivating modes and so accuracy in land use pattern went ahead and the comparing framework about the advancements has been appeared. The preciseness intends to the fine changes in accordance with a wide range of diverse pattern. Furthermore, precise administration measures, to enhance use amount for every single vegetation use, farming material, for example, manure, pesticide, crop variety etc as indicated by the unique state of every cultivating unit, in order to get high return and most monetary advantage, in the interim to decrease the utilization of concoction matter, and to ensure both rural and urban situations and cultivating land asset. The data on product development, the degree of area under cultivations, the effect of watering systems and the change rates of prime pattern and other ecologically delicate grounds and regions suitable for landuse extension is hard to get in effect, it has stayed hard to distinguish the spatial structure and association categorized vegetation land use arranging and administration standards can be utilized..

\section{Study Area}

Kanyakumari is a district in Tamil Nadu state. The total area of the district encompasses over 1672 per sq.km. It spreads over the geographical coordinates between over the eastern longitudes $\left(77^{\circ} 15^{\prime}\right.$ and $\left.77^{\circ} 36^{\prime}\right)$ and northern latitudes $\left(8^{\circ} 03^{\prime}\right.$ and $8^{\circ} 35^{\prime}$.It is the second largest district within the state in terms of population density. The density of populations is 1119 per sq.km and is the highest densely situated populations. The district is surrounded by the ocean on 3 directions and the Western Ghats bordering the northern side.The border of the district expands up toTirunelveli district, the Arabian Sea, the Indian Ocean,state of Kerala, the Gulf of Mannar.The district consists of: Nine taluks: Agastheeswaram, Thovalai, Vilavancode, Kalkulam, Nineblocks:Rajakkamangalam,Thiruvattar,Agastheeswaram,Thovalai, Killiyur, Kurunthancode, Thuckalay, Me lpuram and Munchirai. Four municipalities: Nagercoil,Colachel ,Padmanabhapuram and Andkuzhithurai. Based on topography the area been divided as mountain terrain, plain lands and undulating valley. The district is solely depending on agro based activities and the major income comes from the agriculture. The climatic conditions prevailing in the Kanyakumari district shows distinct variations but on an average moderately suited for all season. This district having rainfall over all months, because of both northeast and southwest monsoon. In October, the northeast monsoon receives an average precipitation of about $549 \mathrm{~mm}$ which starts and terminates in the middle of December. Likewise, the southwest monsoon experience precipitation at a rate of $537 \mathrm{~mm}$, starts from June to September. Relative humidity levels around 60 to 100\%. Rubber, paddy, coconut are the most cultivated crop varieties over the major areas. The well suited topography and climatic conditions lead to the high growth of others yields. 

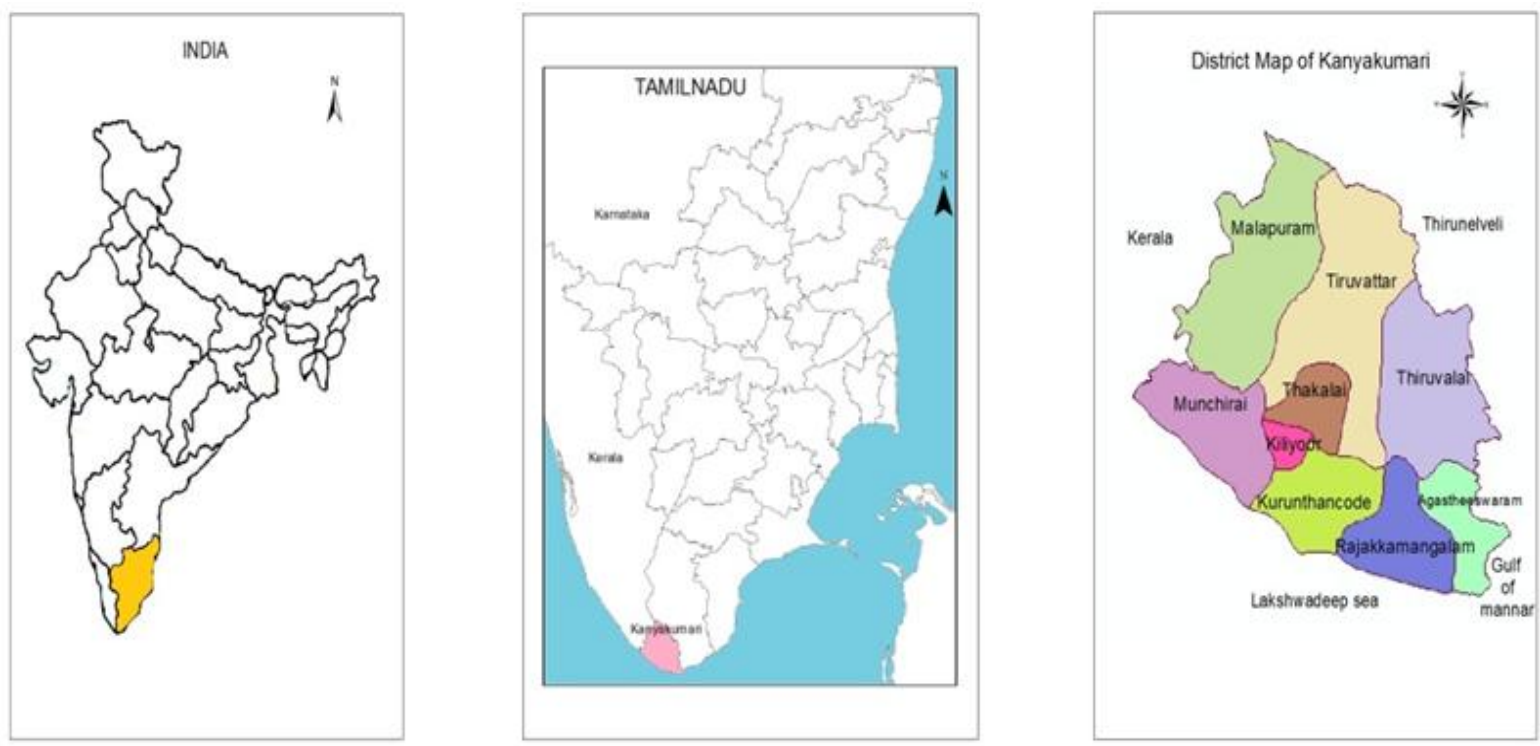

Fig 1: Land-use strategy-Study area

\section{Vegetation Land Use}

The net sown area of the land use is utilized by 80.2 ha. The overall area sown more than once thus occupied by $12.3 \mathrm{ha}$, and overall gross cropped area is about $92.6 \mathrm{ha}$. On the whole the cropping intensity raised around $115.4 \%$. The Kanyakumari accounts for the highest (around 95\%) rubber cultivation in Tamilnadu. Rubber is mainly harvested in the Western Ghats and within the urban, whereas alternative crops and paddy fields extensive measure on the slopes and plains in-between the hills and therefore the coast. The bulk of the crop yields production in the district are: Rubber Paddy, Tapioca, coconut, Clove, Mango, Jackfruit, Pulses, Cashew and Palmyra. Maruthuvalmalai settled among the inexperienced paddy field and coconut palms within the district is acknowledged for the beneficial health plants. This is entirely the district in province with clove plantation Nursing rubber plantations had been tremendously raised in Reserve forest regions in an extremely region of 4785.70 angular distances.

\section{Agro Soil Fertility}

Savvy land-usage includes selecting on learned decisions regarding space use and the natural climatic aspects. Physical and artificial soil data plays a crucial part in the transcription process, reflecting specifically depends on crop yields suitableness and issues regarding the crop yields. The soil in Kanyakumari district is usually composed of red soil selection and along the ocean coasts, variety of soil nearer to the mountain ranges, and gravely available on those areas. Some of the soil types in the district are as Typicdystropepts,Udihapludalfs, Lithicudorthents, Auichapludalfs, Paralithicdystropepts, Parlithicuderthents, Typic mallicdyestopents. Enriched fertile soils are found merely at Thiruvattar, then at Killiyoor, next to that Munchirai, Rajakamanagalam and Thuckalay blocks. In Agastheeswaram taluk and in Thovalai Red soils are found. The $\mathrm{pH}$ scale ranges between 4.5 to 8.0.For enriched soil -potassium extends to high range around $49 \%$ of the land within the district is beneath cultivation and around $31 \%$ is roofed by forest, nitrogen extends from low range to medium level, phosphorus content in medium level.

\section{Data Source}

It has been noted over time through series of studies that the Landsat Thematic clerk is adequate for general intensive synoptic coverage of huge areas.It comprises of seven spectral bands .Here we tend to do region unit exploitation of the Landsat picture from USGS Earth. the information is in GeoTiff design with sixteen piece radiometric determination (ranges from 0-65535). As a result, this reduces the necessity for pricey and time-over whelming ground surveys conducted for validation of knowledge. The land cover area wise statistical data been gathered from the Tamilnadu government statistical analysis of Kanyakumari district. Generally, satellite imagination is in a position so as to produce additional frequent information assortment on an everyday basis. Approximation of the agro-land-cover data, natural variations, plant growth situations just like calculating Vegetation Index (NDVI) is done. The transformations are helpful measures of vegetation calculated from the remotely detected information. This method is comparatively right forward to figure out these indices mistreatment information from the Landsat sensors and ArcMap. 
Table 1- Quantitative Data: Area wise classification

\begin{tabular}{|l|l|l|l|l|}
\hline Classification & Thiruvattar & Munchirai & Melpuram & Kiliyoor \\
\hline Reserve Forest & 19704 & 0 & 11696 & 0 \\
\hline Uncultivable uses & 241 & 33 & 91 & 136 \\
\hline Non-agricultural uses & 2081 & 1416 & 3107 & 1731 \\
\hline Fallow land & 25 & 129 & 306 & 214 \\
\hline Net area sown & 12346 & 5370 & 11509 & 6196 \\
\hline Total geographic area & 34480 & 7201 & 27189 & 8785 \\
\hline
\end{tabular}

\begin{tabular}{|l|l|l|l|l|l|}
\hline Classification & Agastheeswaram & Rajakamangalam & Thovalai & Kurunthancode & Tukalay \\
\hline Reserve Forest & 795 & 0 & 20856 & 813 & 291 \\
\hline Uncultivable uses & 535 & 463 & 1658 & 135 & 709 \\
\hline Non-agro use & 4835 & 4575 & 5637 & 2513 & 3288 \\
\hline Fallow land & 105 & 101 & 267 & 101 & 39 \\
\hline Net area sown & 7491 & 8207 & 8015 & 7424 & 9330 \\
\hline Total-geographic area & 14182 & 13573 & 36907 & 11203 & 13680 \\
\hline
\end{tabular}

Table 2: Crop Productions

\begin{tabular}{|l|l|l|l|}
\hline \multicolumn{1}{|c|}{ Crops } & Area(hec) & Production $\mathrm{Kg}$ & Average yield rate $(\mathrm{Kg} / \mathrm{hec})$ \\
\hline Paddy Kar & 7023 & 32411145 & 4615 \\
\hline Paddy Samba & 6596 & 34292604 & 5199 \\
\hline Pulses & 737 & 627924 & 852 \\
\hline Groundnut & 20 & 44260 & 2213 \\
\hline Coconut & 23917 & 287099668 & 12004 \\
\hline Banana & 6238 & 188967734 & 30293 \\
\hline Mango & 1422 & 8246178 & 5799 \\
\hline Jack & 630 & 14992110 & 23797 \\
\hline Cashews & 1174 & 334590 & 285 \\
\hline Tapioca & 2887 & 100969938 & 34974 \\
\hline Tamarind & 1067 & 2075315 & 1945 \\
\hline Other crop rubber & 27407 & 85117200 & 4200 \\
\hline
\end{tabular}

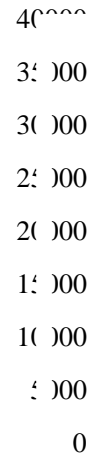

Total

Geographi

cal

Area(ha)

Net Area

Sown(ha)

Gross

Cropped

Area(ha)

Fig 2: Quantitative Land representation

\section{Integrating With Gis}

The Geographical Information System(GIS) thus employed for describing the special attribute variations of aerial classification.GIS process the geographical data, disposal, analysis, the graph conversion and model integrating for the spatial attribute knowledge. Various ground truth attribute data had been collected for years and mapped according to that. Arc Map and ArcGIS professional will offer a magnificent user expertise in virtualized environments. Arc-Map enables GIS processing, formatting, editing with proper visualization, and geo-processing application that permits us to form maps, and manage spatial information to perform the total spectrum of analyses required to replicate the related data into valuable information in an understandable view. For the study regarding land use pattern .It is necessary to a certain data regarding to time relative property under continuous comparability, and high spatial resolution is required within the study region. 


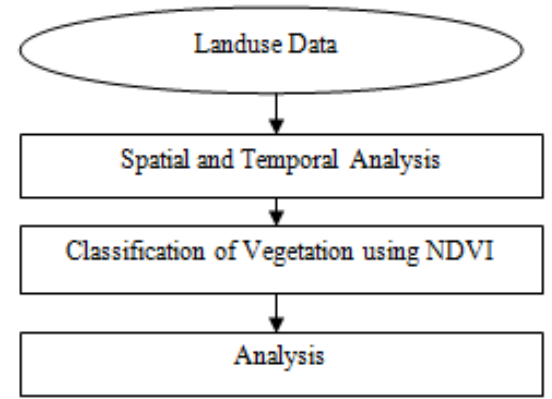

Fig 3: Flow Chart - GIS implementation

\section{Analysis}

In order to acknowledge the analyzed vegetation stress modification, Landsat satellite imaging of Kanyakumari were accustomed and Normalized Difference Vegetation Index (NDVI) is calculated.

- Utilizing Arc catalogue toolbars for the evaluation of Vegetation index with the relevant band cited for raster calculation.

- Landsat 7 Operational Land Imager and Thermal Infrared finder pictures envelop 9 phantom groups with a thirty meter resolution for Bands one to seven and nine. The resolution regarding Band nine, it is fifteen meters which is panchromatic. Furthermore it even has 2 Thermal IR groups with a resolution of 100meters (later re-sampled into thirty meters).

- Before hard the NDVI, the Digital Number data ought to be recover to coefficient of reflection exploitation the mathematical statements given in their site. Here the IR and NIR groups region unit four and five severally. Its associate degree formula designed to differentiate the inexperienced vegetation from different surfaces supported the vegetation coefficient properties of the world.

- The results analysis of the fields regarding to NDVI worth are between -1 and one. NDVI values larger than zero indicate the presence of vegetation.

- The higher the vegetation index, denser the vegetation. While, negative values indicate no vegetation and correspond to watershed coverage.

- The ultimate NDVI maps were classified into four classes. That's the water bodies $(<0)$, extremely stressed (0to0.2) moderately stressed ( 0.2 to 0.4$)$ and low stressed areas (NDVI $\geq 0.4$ ).

- For reference of analyzing the land use at particular year 2015, we make a comparison with 2010 to determine the total changeover occurrences.

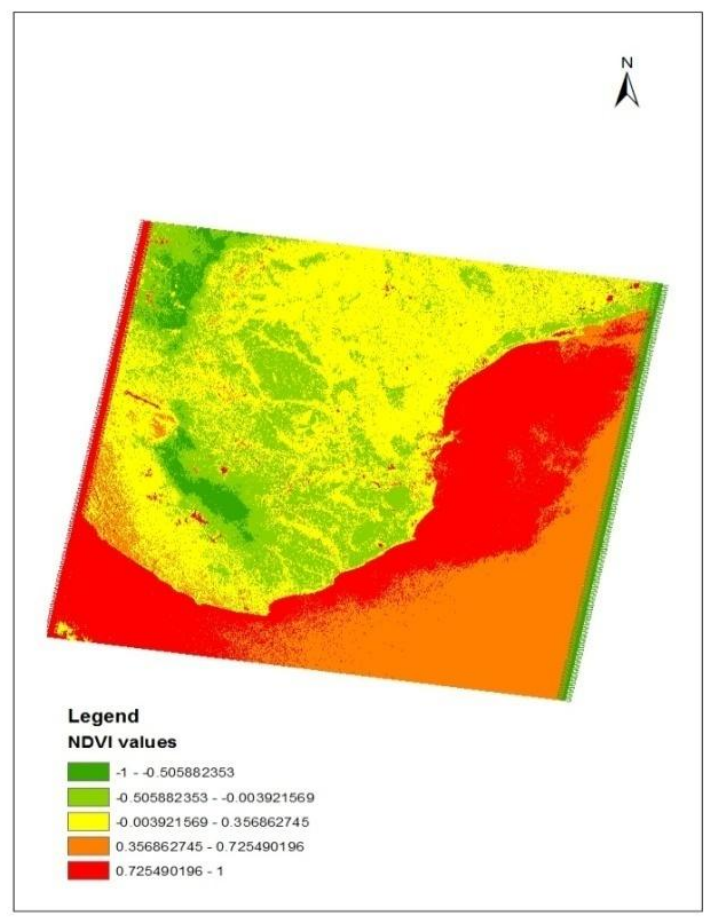

Fig 4: NDVI Landuse Pattern(2010) 


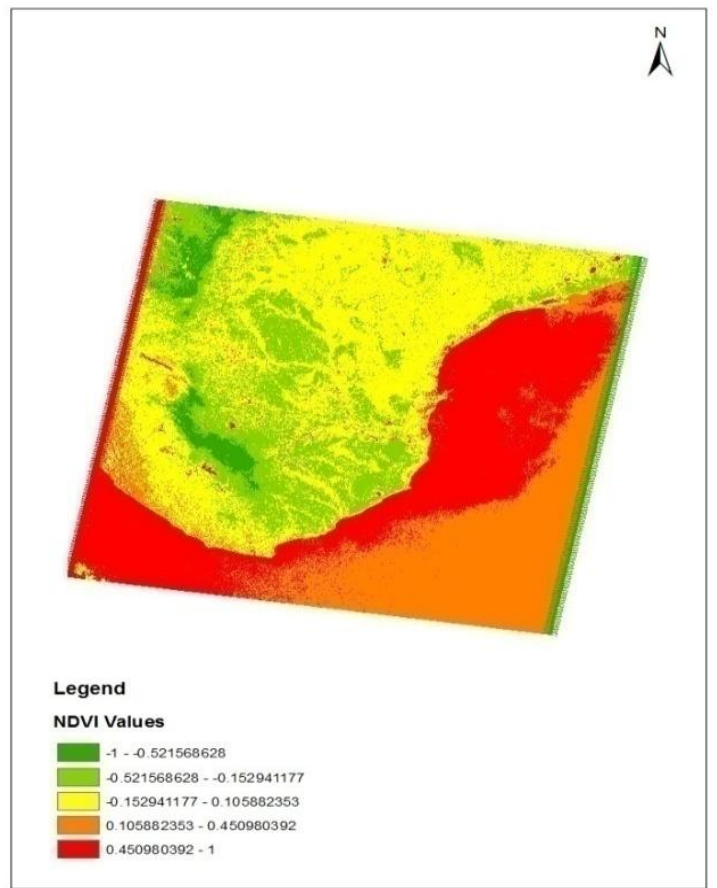

Fig 5: NDVI Landuse Pattern(2015)
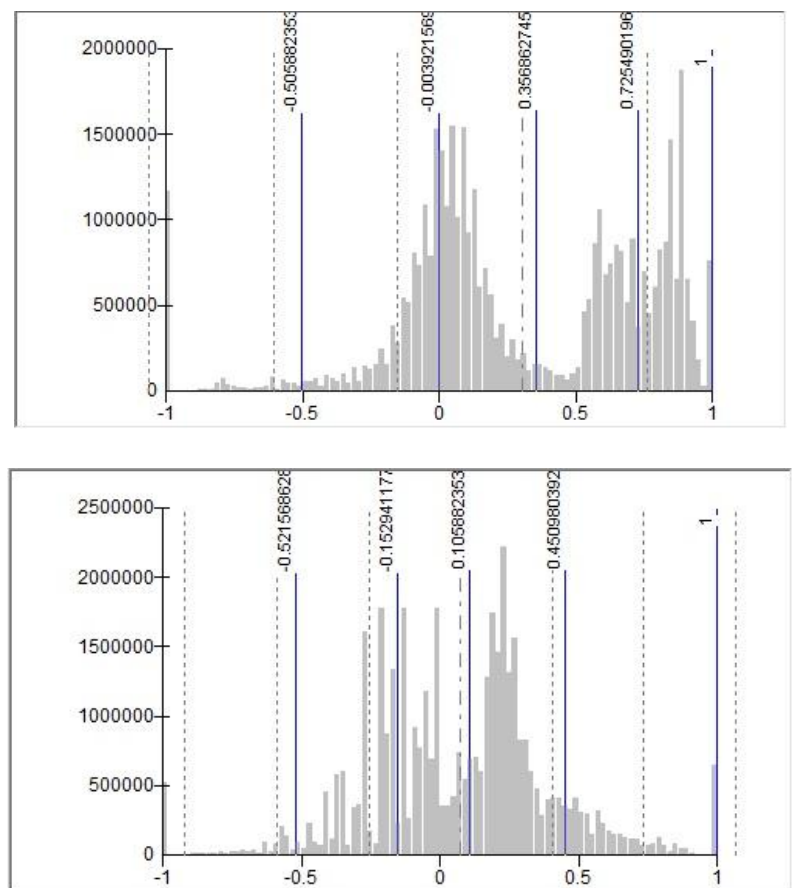

Fig 6: Histogram Analysis: NDVI (2010 and 2015)

Standard Deviation

Histogram intensity

- . . . . - Mean

\section{Results And Inferences}

The analyzed satellite images and maps obtained thus pre-processed under supervised and significant classification shows the land use of the study area as given in the figures. These images offer the knowledge about the land use pattern of the study space. The dark yellow-color belongs to the urban settled space; Green color resembles the agricultural/vegetation crop yields over the realm. Therefore, with relevant water resources could improve higher cultivation. As inferred from the NDVI values the 2015 vegetation space had 
been increased as compared to past years. Area under yellow and green color resembles land use. Therefore at massive spatial diversities, the inter-annual changes of NDVI values, as computed for instance by the quality variation of most NDVI, could reflect variable and unexpected environments. Consequently, maximum values thus belong to normal deviation and constants of variations to have a negative relationship with some of the species richness. The NDVI analysis of vegetation resembles the usage and density of high crop yields over the realm.

\section{Conclusion}

Thus the work had been carried out for the landuse pattern analysis in Kanyakumari District. The analysis had been carried out with the NDVI classification of the respective study area .As such the entire land use had been visualized with the help of ArcGIS mapping software. Land use is the most observable of all environmental changes and assessment of any changes in ecosystem can be observed through the analysis. Further work will be expanded upon all the states in India

\section{References}

[1]. Wei Hong Liu, Yi Nan Zhang, Feng Xia Wang, Land Use Changes Of Xiangshan County Based On RS And GIS,

[2]. The 2014 7th International Congress on Image and Signal Processing

[3]. Usha A, Edwin Gnanadhas M. ,A study on sustainable tourism development in KanyakumariDistrict. ISSN 2014

[4]. Z. Ren, Y. Shan, and Y. Wang, Study and application on investigation and grading of land fertility of standard farmland in Zhejiang Province ,ActaAgriculturaeZhejiangensis, vol. 23, no. 2, pp. 404-408, March 2011

[5]. Punithavathi J, Tamilenthi S, Baskaran R,Agricultural concentration and crop wise changes in Thanjavur District, Tamil Nadu using geographical information system, International Multidisciplinary Research Journal. ISSN: 2231-6302. 2012; 2(7):44-8.

[6]. L. Teng, Y. Hua, and Y. Tong,Design and Realization of a High Quality Prime Farmland Management Information System in Zhejiang Province, Journal of Jiangnan University(Natural Science Edition), vol. 9, no. 1, pp. 35-38, January 2010

[7]. Yulin Prefecture, Zhongfeng Li, XuemeiLi,Remote Sensing and GIS Application for Agricultural Land Quality Assessment,Geography\& Tourism College, Chongqing Normal University, Chongqing, 400047, China

[8]. Balqies Sadoun and Samih Al Rawashdeh ,Applications of GIS and Remote Sensing Techniques to Land Use Management, 978-14244-3806-8/09/\$25.00 @ 2009 IEEE

[9]. Y. Chen and D. Gillieson ,Evaluation of Landsat TM vegetation indices for estimating vegetation cover on semi-arid rangelands-A case study from Australia, Remote Sens., vol. 35, no. 5, pp. 435-446, Oct. 2009

[10]. Michel A Mulders, Advances in the application of remote sensing and GIS for surveying mountainous land, International Journal of Applied Earth Observation and Geoinformation, 2001,3(1),3-1O.

[11]. Government of Tamilnadu, Kanyakumari agriculture and irrigation department

[12]. C.L.GU., Sinica, Beijing land use / cover change mechanism, Natural resources , 1999, 14 (4): $307-312$.

[13]. Green.K,D.Kemka ,L.Lackey,Using Remote Sensing to Detect and Monitor Land-cover and Land use Change, 1994. PE \& RS Vol.60(3):331-337

[14]. W. Xiaodong and C. Weihong,Effect of Dynamic Monitoring Landuse/Landcover, Territory \& Natural Resources Study, no. 3, pp. 27-31, 1998

[15]. Lucas L. F. Janssen and Martien Molenaar, Terrain Objects their Dynamics and their Monitoring by the Integration of GIS and Remote Sensing, IEEE transactions on geoscience and remote sensing, vol. 33, no. 3, may 1995 